\title{
Correction to: Triplanar correction of adolescent idiopathic scoliosis by asymmetrically shaped and simultaneously applied rods associated with direct vertebral rotation: clinical and radiological analysis of 36 patients
}

\author{
Cesare Faldini ${ }^{1}\left[\right.$ [ Fabrizio Perna ${ }^{1} \cdot$ Giuseppe Geraci ${ }^{1} \cdot$ Francesco Pardo $^{1} \cdot$ Antonio Mazzotti $^{1} \cdot$ Federico Pilla ${ }^{1}$.
} Alberto Ruffilli ${ }^{1}$

Published online: 31 October 2018

(c) Springer-Verlag GmbH Germany, part of Springer Nature 2018

\section{Correction to: \\ European Spine Journal (2018) 27 (Suppl 2):S165-S174 \\ https://doi.org/10.1007/s00586-018-5595-z}

Unfortunately, the affiliation of the author group has been incorrectly published in original version. The complete correct affiliation of all authors should read as follows.

IRCCS Istituto Ortopedico Rizzoli, Via Giulio Cesare Pupilli 1, 40136, Bologna, Italy

The original article can be found online at https://doi.org/10.1007/ s00586-018-5595-z.

Cesare Faldini

cesare.faldini@ior.it

1 IRCCS Istituto Ortopedico Rizzoli, Via Giulio Cesare Pupilli 1, 40136 Bologna, Italy 